\title{
Nutrição parenteral no recém-nascido pré-termo: proposta de protocolo prático
}

\author{
Parenteral nutrition in preterm infants: proposal of a practical guideline
}

Fabíola Isabel S. de Souza', Márcia Teske², Roseli Oselka S. Sarni

\section{RESUMO}

Objetivo: Revisar a literatura e os conceitos relacionados à terapia nutricional parenteral de recém-nascidos pré-termo e propor fluxograma prático de indicação, progressão dos parâmetros e monitoramento para utilização em unidades neonatais.

Fontes de dados: Artigos publicados em inglês e português nas bases de dados Medline, Embase, Lilacs e SciELO nos últimos dez anos, além de referências clássicas e consensos internacionais. As palavras-chave utilizadas como fonte de busca foram recém-nascido pré-termo, nutrição parenteral, terapia nutricional e emulsões lipídicas.

Síntese dos dados: A nutrição parenteral é procedimento essencial no tratamento intra-hospitalar do recém-nascido pré-termo. Além dos avanços no conhecimento e progressos na legislação, vários fatores contribuíram para reduzir a morbimortalidade desses recém-nascidos e elevar a segurança na utilização da nutrição parenteral, tais como qualidade dos cateteres empregados, treinamento e capacitação adequada dos profissionais envolvidos, existência de equipes multiprofissionais e o desenvolvimento de novos insumos.

Conclusões: Esse protocolo prático sobre nutrição parenteral para recém-nascidos prematuros foi desenvolvido com base em recomendações internacionais de sociedades científicas e na análise crítica de estudos científicos.

Palavras-chave: nutrição parenteral; recém-nascido; prematuro; recomendações nutricionais.

\section{ABSTRACT}

Objective: Review the literature regarding parenteral nutrition of preterm infants in order to propose a practical guideline for indication, increase of parameters and monitoring of this nutritional therapy in neonatal units.

Data source: Studies in English and Portuguese from the last ten years were retrieved from Medline, Embase, Lilacs and SciELO using the following key-words: preterm infants, parenteral nutrition, nutrition therapy and lipid emulsions. Also classical studies and consensus on the theme were manually searched.

Data synthesis: Parenteral nutrition is an essential treatment strategy for preterm infants. Besides progress in knowledge and legislation, several factors contribute to reduce neonatal morbidity and mortality of newborns using parenteral nutrition and to increase the security in its prescription such as catheters' quality, training of the multiprofessional team and development of new specific parenteral nutrition formulations.

Conclusions: The practical parenteral nutrition guideline proposed here follows international guidelines and was based on critical analysis of the studies published in the last 10 years.

Key-words: parenteral nutrition; infant newborn; infant premature; nutrition policy.
${ }^{1}$ Mestre em Ciências pela Escola Paulista de Medicina da Universidade Federal de São Paulo (Unifesp-EPM), médica colaboradora do Serviço de Nutrologia do Departamento de Pediatria da Faculdade de Medicina do $A B C$ (FMABC) e coordenadora clínica da Equipe Multidisciplinar de Terapia Nutricional do Hospital Estadual Mário Covas e Hospital Municipal Universitário de São Bernardo do Campo da Fundação do ABC. Santo André, SP, Brasil

${ }^{2}$ Médica colaboradora do Serviço de Nutrologia do Departamento de Pediatria da FMABC. Santo André, SP, Brasil

${ }^{3}$ Doutora em Medicina e médica assistente do Departamento de Pediatria da Unifesp-EPM, professora-assistente do Departamento de Pediatria e coordenadora do Serviço de Nutrologia do Departamento de Pediatria da FMABC. Santo André, SP, Brasil
Endereço para correspondência:

Roseli Oselka Saccardo Sarni

Rua René Zamlutti, 94 - apto. 52 - Vila Mariana

CEP 04116260 - São Paulo/SP

E-mail: rssarni@unifesp.br / rssarni@uol.com.br

Recebido em: 7/1/2008

Aprovado em: 10/4/2008 


\section{Introdução}

A nutrição parenteral (NP) é procedimento terapêutico essencial no tratamento intra-hospitalar do recém-nascido pré-termo $(\mathrm{RNPT})^{(1)}$. A terapia nutricional do RNPT representa um enorme desafio, pois quanto menor o peso e a idade gestacional do recém-nascido $(\mathrm{RN})$, maiores serão as suas necessidades nutricionais para atingir crescimento e desenvolvimento adequados ${ }^{(2,3)}$. Os RNPT têm reservas reduzidas e maior imaturidade do trato gastrintestinal e de outros órgãos, sistemas e aparelhos, o que impossibilita a utilização da via enteral de forma exclusiva, especialmente nas primeiras semanas de vida $^{(4-6)}$. Assim, os avanços nos procedimentos relacionados à NP, tais como o tipo de cateter utilizado ${ }^{(7,8)}, \mathrm{o}$ treinamento e capacitação dos profissionais envolvidos ${ }^{(9)}$ e o desenvolvimento de novos insumos, aliados ${ }^{(10)}$ aos progressos na legislação ${ }^{(11,12)}$, têm contribuído sobremaneira para elevar a segurança na utilização desta forma de terapia nutricional.

A NP é um procedimento que faz parte da rotina da assistência de terapia intensiva neonatal, sendo importante desenvolver protocolos práticos que levem em conta os avanços disponíveis na literatura e propostas de guias de conduta internacionais. Nesse contexto, o objetivo deste artigo é, com uma revisão adequada da literatura, apresentar os conceitos relacionados à terapia nutricional parenteral de RNPT e propor um protocolo prático de indicação, progressão dos parâmetros e monitoramento, que possa ser utilizado no treinamento de profissionais, como residentes de pediatria e equipes multiprofissionais que atuam com terapia nutricional neonatal.

Para fazer a revisão de literatura, foram utilizados as diretrizes propostas por diversas sociedades científicas internacionais como American Academy of Pediatrics (APA) ${ }^{(13)}$, American Society for Parenteral e Enteral Nutrition (Aspen) $)^{(14)}$ e European Society for Clinical Nutrition and Metabolism (Espen) e European Society for Pediatric Gastroenterology Hepatology and Nutrition (ESPGHAN) $)^{(15)}$ e por artigos em inglês e português relacionados a terapia nutricional parenteral de RNPT, publicados nas bases de dados Medline, Embase, Lilacs e SciELO, nos últimos dez anos. As palavras-chave utilizadas como fonte de busca foram: recém-nascido pré-termo, nutrição parenteral, terapia nutricional, emulsões lipídicas.

\section{Legislação}

O Brasil conta com legislação específica desde 1998. Tratase da Portaria $n^{\circ}$ 272/MS/SNVS, de 8 de abril de $1998^{(11)}$, que fixa os requisitos mínimos exigidos para a terapia de nutrição parenteral, normatizando a indicação, a prescrição, a preparação farmacêutica (avaliação farmacêutica, manipulação, controle de qualidade, conservação e transporte), a administração, o controle clínico e laboratorial.

Em 2005, o Ministério da Saúde publicou uma série de portarias que instituiu, no âmbito do Sistema Único de Saúde (SUS), a Alta Complexidade em Terapia Nutricional (Unidades de Assistência e os Centros de Referência) ${ }^{(12)}$. Nestas portarias estão incluídos diversos procedimentos de terapia nutricional para adultos e crianças, incluindo a nutrição parenteral neonatal, o que representou grande avanço da legislação no reconhecimento deste procedimento terapêutico.

\section{Indicação}

Quanto menor o RNPT, mais precoce deve ser a indicação da NP, uma vez que as reservas energéticas destas crianças são bastante limitadas ${ }^{(1,16)}$. Estima-se que um RN de $1.000 \mathrm{~g}$ teria reservas energéticas suficientes para sobreviver, sem terapia nutricional, por apenas quatro dias ${ }^{(17,18)}$.

Crianças com peso inferior a $1000 \mathrm{~g}$ devem receber NP nas primeiras horas de vida e sugere-se, inicialmente, solução contendo aminoácidos, glicose e eletrólitos ${ }^{(18,19)}$. RNPT com 1000 a $1500 \mathrm{~g}$ também merecem atenção especial, não devendo ser postergado o início da NP por mais do que 48 horas. Mesmo no $\mathrm{RN}$ com peso superior a $1500 \mathrm{~g}$, estes não devem permanecer mais de 72 horas sem terapia nutricional efetiva ${ }^{(1)}$ (Figura 1 ).

A nutrição enteral deve ser administrada, preferencialmente, com leite humano e ainda o mais precocemente possível (nas primeiras 48 horas de vida), mesmo em pequenos volumes (Figura 1), por representar potente fator de estímulo para maturação do trato gastrintestinal e proteção contra infecções, além de outras vantagens ${ }^{(20-22)}$.

No Brasil, assim como em países europeus, utilizam-se soluções 3:1 (dextrose, lipídios e proteínas no mesmo recipiente). Em alguns países, como os Estados Unidos, o lipídio é ofertado em via separada dos demais nutrientes, visando reduzir os riscos de incompatibilidades e precipitação.

A seguir, serão abordados de maneira individualizada as etapas e os diferentes componentes da NP a fim de formular um protocolo prático para utilização da NP em RNPT. Sugere-se a leitura combinada com a observação da Figura 1.

\section{Acesso venoso}

Soluções de NP com osmolaridade acima de $600 \mathrm{mOsm} / \mathrm{L}$ (Quadro 1), utilizadas em acessos periféricos, podem levar 


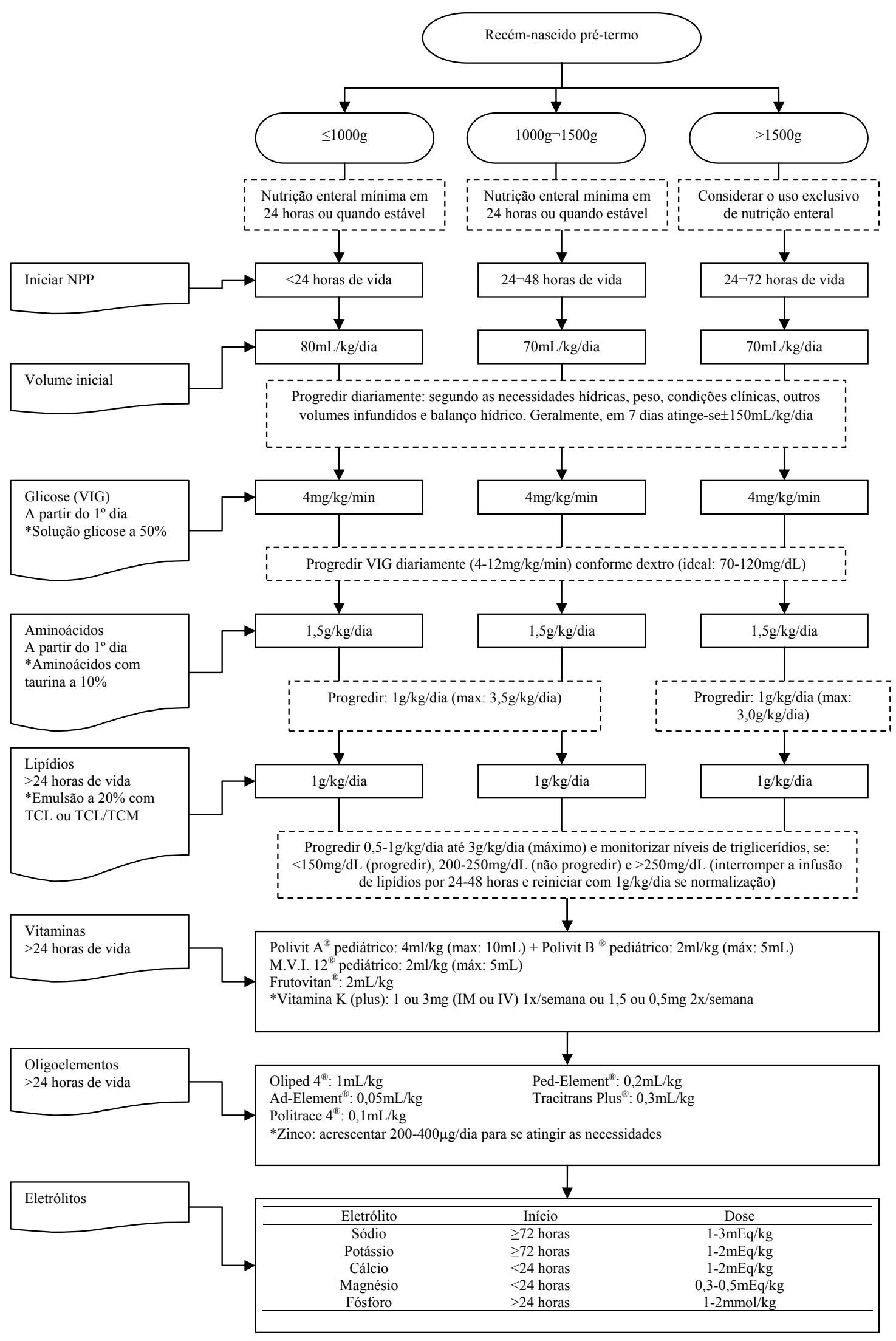

Figura 1 - Algoritmo de indicação e seguimento da terapia nutricional parenteral de recém-nascido pré-termo. 
a uma série de complicações (flebite, extravasamento etc). Como, na maioria das vezes, a NP do RNPT excede tal valor e o seu emprego ocorre por período prolongado ( $>14$ dias), prefere-se, já inicialmente, contar com um acesso central.

A utilização de cateteres centrais de inserção periférica peripherally inserted central catheters (PICCs) representou uma verdadeira revolução na escolha da via de acesso para NP pela sua segurança e facilidade de inserção. Atualmente, tais cateteres são considerados como primeira opção para infusão de NP em neonatos ${ }^{(7)}$. A infusão de NP por cateteres umbilicais está relacionada a maior número de complicações mecânicas e infecciosas, devendo ser utilizada somente em situações de exceção e por período curto (artéria $<5$ dias e a veia $<14$ dias) ${ }^{(23)}$. Assim, o cateter em veia umbilical pode ser empregado nas primeiras horas de vida, enquanto é providenciada a inserção do PICC.

\section{Aminoácidos}

Estudos mostram que a infusão inicial de $1,5 \mathrm{~g} / \mathrm{kg} / \mathrm{dia}$ de aminoácidos, seguida de aumentos de 0,5 a $1 \mathrm{~g} / \mathrm{kg} / \mathrm{dia}$ até 3 a $3,5 \mathrm{~g} / \mathrm{kg} / \mathrm{dia}$, é necessária para promover balanço nitrogenado positivo em $\mathrm{RNPT}^{(23,24)}$. A infusão de taxas maiores de aminoácidos ( 3 a $3,5 \mathrm{~g} / \mathrm{kg} / \mathrm{dia}$ ) nas primeiras horas de vida, mesmo em $\mathrm{RN}$ com peso $<1000 \mathrm{~g}$, não induz à toxidade e promove melhor balanço nitrogenado imediato, quando comparado a taxas menores ( 1 a $1,5 \mathrm{~g} / \mathrm{kg} / \mathrm{dia})^{(13,25,26)}$, entretanto ainda não há trabalhos avaliando eventos adversos de tal prática em longo prazo $^{(27)}$. No presente protocolo, preconizou-se iniciar com $1,5 \mathrm{~g} / \mathrm{kg} / \mathrm{dia}$ e promover incrementos rápidos $(1 \mathrm{~g} / \mathrm{kg} /$ dia e máximo de $4 \mathrm{~g} / \mathrm{kg} / \mathrm{dia})$, o que permite atingir em 72 horas a oferta proposta (Figura 1).

A oferta protéica em fases iniciais da NP em RNPT é muito superior à preconizada para crianças maiores em diferentes situações clínicas. Sendo assim, o cálculo da relação gramas de nitrogênio/calorias não protéicas não deve ser valorizado nessa fase do planejamento da $\mathrm{NP}^{(19,24)}$. O metabolismo energético do RNPT é diferente, especialmente naqueles $<1000 \mathrm{~g}$, com produção de glicose a partir de neoglicogênese e uso de proteína como substrato energético, mesmo em condições de estabilidade clínica ${ }^{(24,28)}$.

Alguns aminoácidos são considerados condicionalmente essenciais para os RNPT e, por isso, a escolha de soluções pediátricas é fundamental para garantir segurança e atender às necessidades nutricionais específicas. Tais soluções, especialmente adaptadas, contêm taurina, tirosina, histidina, ácido aspártico e ácido glutâmico em quantidades semelhantes às encontradas no leite humano, mas contém menor concentração de metionina, glicina e fenilalanina ${ }^{(13,29)}$ (Tabela 1).
A cisteína, também é um aminoácido condicionalmente essencial no período neonatal, especialmente para aqueles com menos de 32 semanas $^{(30)}$. Esse aminoácido é o maior substrato para a síntese do tripeptídeo glutationa (parte integrante da enzima glutationa peroxidase, com função antioxidante) e não faz parte das soluções de aminoácidos devido à baixa estabilidade. Entretanto, a cisteína pode ser adicionada à solução de NP (30 a 55mg/ $\mathrm{kg} / \mathrm{dia}$ ou 200 a $350 \mu \mathrm{mol} / \mathrm{kg}$ ou $40 \mathrm{mg} / \mathrm{g}$ proteína na solução) para garantir a normalização dos níveis plasmásticos, especialmente se a criança não está recebendo nutrição enteral com leite humano ${ }^{(13,29)}$.

Em relação à glutamina, não há evidências atuais de benefícios com o seu uso em RNPT ${ }^{(13,14,30,31)}$. Se administrada, não se deve ultrapassar 20\% da quantidade de aminoácidos infundida $(0,4 \text { a } 0,5 \mathrm{~g} / \mathrm{kg} / \mathrm{dia})^{(14,29)}$.

A carnitina, utilizada no transporte de ácidos graxos de cadeia longa através da membrana mitocondrial, também não está presente nas soluções de aminoácidos atualmente disponíveis. Sua suplementação deve ser considerada em crianças recebendo NP de forma exclusiva por período superior a quatro semanas. A dose sugerida por via parenteral é de $10 \mathrm{mg} / \mathrm{kg} / \mathrm{dia}^{(29)}$.

\section{Lipídios}

A infusão de lipídios na NP do RNPT é importante para fornecer energia e ácidos graxos essenciais, sendo a recomendação de ácido linoléico para o RNPT de 0,25g/ dia, podendo ser iniciada precocemente ${ }^{(32,33)}$ e com tolerância melhorada se a infusão ocorrer de forma contínua, no período das 24 horas $^{(34)}$.

De forma prática, a infusão lipídica pode ser iniciada a partir do segundo dia de vida ( $>24$ horas de vida), com taxa de infusão inicial de 0,5 a $1 \mathrm{~g} / \mathrm{kg} / \mathrm{dia}$ (máximo de $4 \mathrm{~g} / \mathrm{kg} / \mathrm{dia}$ ) e incrementos de 0,5 a $1 \mathrm{~g} / \mathrm{kg} / \mathrm{dia}$. Porém, atenção especial deve ser dada à monitorização dos triglicerídios séricos, sendo necessária nova dosagem a cada aumento de $1 \mathrm{~g} / \mathrm{kg}^{(34)}$ (Quadro 2). Sugerese interromper a progressão da infusão lipídica quando os níveis de triglicérides estiverem acima de $200 \mathrm{mg} / \mathrm{dL}$ e suspendê-la se acima de $250 \mathrm{mg} / \mathrm{dL}$, retornando após 24 a 48 horas com $1 \mathrm{~g} / \mathrm{kg}$, dependendo da condição clínica. Outro fato importante a ser observado é que a taxa de infusão de lipídios não deve exceder 0,13 a $0,17 \mathrm{~g} / \mathrm{kg} / \mathrm{h}^{(34)}$ (Figura 1 ). Diversos fatores, além da taxa de infusão de lipídios, influenciam a trigliceridemia, entre eles a prematuridade extrema, a sepse, a colestase, o catabolismo, a insuficiência renal e a pancreatite, entre outros. 
Tabela 1 - Composição das soluções de aminoácidos cristalinos para uso parenteral

\begin{tabular}{|c|c|c|c|c|}
\hline Composição & Travasol ${ }^{\circledR} 10 \%$ & Aminosteril $^{\otimes} 10 \%$ & Primene $^{\circledR} 10 \%$ & Aminoped $^{\circledR} 10 \%$ \\
\hline$(\mathrm{g} / 100 \mathrm{~mL})$ & Baxter & Fresenius Kabi & Baxter & Fresenius Kabi \\
\hline Leucina & 0,73 & 0,74 & 1,00 & 1,07 \\
\hline Fenilalanina & 0,56 & 0,51 & 0,42 & 0,46 \\
\hline Lisina & 0,58 & 0,66 & 1,10 & 0,71 \\
\hline Metionina & 0,40 & 0,43 & 0,24 & 0,46 \\
\hline Isoleucina & 0,60 & 0,50 & 0,67 & 0,64 \\
\hline Valina & 0,58 & 0,62 & 0,76 & 0,71 \\
\hline Histidina & 0,48 & 0,30 & 0,38 & 0,41 \\
\hline Treonina & 0,42 & 0,44 & 0,37 & 0,52 \\
\hline Triptofano & 0,18 & 0,20 & 0,20 & 0,18 \\
\hline Alanina & 2,07 & 1,50 & 0,80 & 0,72 \\
\hline Glicina & 1,03 & 1,40 & 0,40 & -- \\
\hline Arginina & 1,15 & 1,12 & 0,84 & 0,64 \\
\hline Prolina & 0,68 & 1,50 & 0,30 & 1,61 \\
\hline Serina & 0,50 & -- & 0,40 & 0,90 \\
\hline Tirosina & 0,04 & -- & 0,05 & 0,55 \\
\hline Cisteína & -- & -- & 0,19 & 0,04 \\
\hline Ácido aspártico & -- & -- & 0,60 & -- \\
\hline Ácido glutâmico & -- & -- & 1,00 & -- \\
\hline Ornitina & -- & -- & 0,25 & -- \\
\hline Taurina & -- & -- & 0,06 & -- \\
\hline Osmolaridade-mOsm/L & 998 & 939 & 790 & 848 \\
\hline
\end{tabular}

Travasol $^{\circledR}$ e Aminosteri ${ }^{\circledR}=$ são soluções padrão; Primene $^{\circledR}$ e Aminoped $^{\circledR}=$ são soluções pediátricas, sendo que a última vem com taurina (aminoácido condicionalmente essencial para RNPT).

Estudos recentes mostraram que a utilização de heparina na NP não melhora o clareamento lipídico e por isso, atualmente, não se recomenda sua utilização rotineira nas soluções com esta finalidade. Em algumas situações, o emprego de heparina pode ser benéfico por aumentar o tempo de duração dos cateteres e também por reduzir o risco de obstrução ${ }^{(35)}$. A dose sugerida é $0,5 \mathrm{U} / \mathrm{mL}$ da solução de $\mathrm{NP}$, lembrando que sua adição eleva o risco de instabilidade na solução de NP 3:1.

Enormes avanços aconteceram nos últimos anos em relação à composição das emulsões lipídicas para NP. Atualmente, há no mercado emulsões com ácidos graxos de cadeia longa e média, somente de cadeia longa (óleo de soja), emulsões com óleo de oliva, óleo de peixe e lipídios estruturados (óleo de soja, oliva, peixe e triglicérides de cadeia média). Entretanto, há poucos estudos utilizando estas novas emulsões lipídicas na NP do RNPT, o que, infelizmente, limita o seu uso corrente ${ }^{(33)}$ (Tabela 2).
Na prática, preferir emulsões lipídicas a 20\%, uma vez que sua menor relação entre fosfolipídios e triglicerídios promove um melhor clareamento e, consequentemente, menor risco de hipertrigliceridemia, em comparação às emulsões a $10 \%^{(34)}$. É possível utilizar emulsões contendo somente triglicéride de cadeia longa ou meio a meio com triglicérides de cadeia média e longa. Os últimos, apesar de terem menor conteúdo de ácidos graxos essenciais, teoricamente são metabolizados mais rapidamente por ser o triglicéride de cadeia média praticamente independente da carnitina para entrar na mitocôndria e posterior oxidação ${ }^{(33,34)}$.

Não há evidente associação entre hiperbilirrubinemia, trombocitopenia e colestase com a infusão de lipídios, entretanto, se forem afastadas todas as outras causas relacionadas a estas doenças, bastante comuns no RNPT, deve-se monitorizar com mais rigor os níveis de triglicerídios. Em última instância, na trombocitopenia e colestase, pode-se reduzir ou suspender temporariamente ( 24 a 
Tabela 2 - Composição das emulsões lipídicas tradicionais para uso parenteral

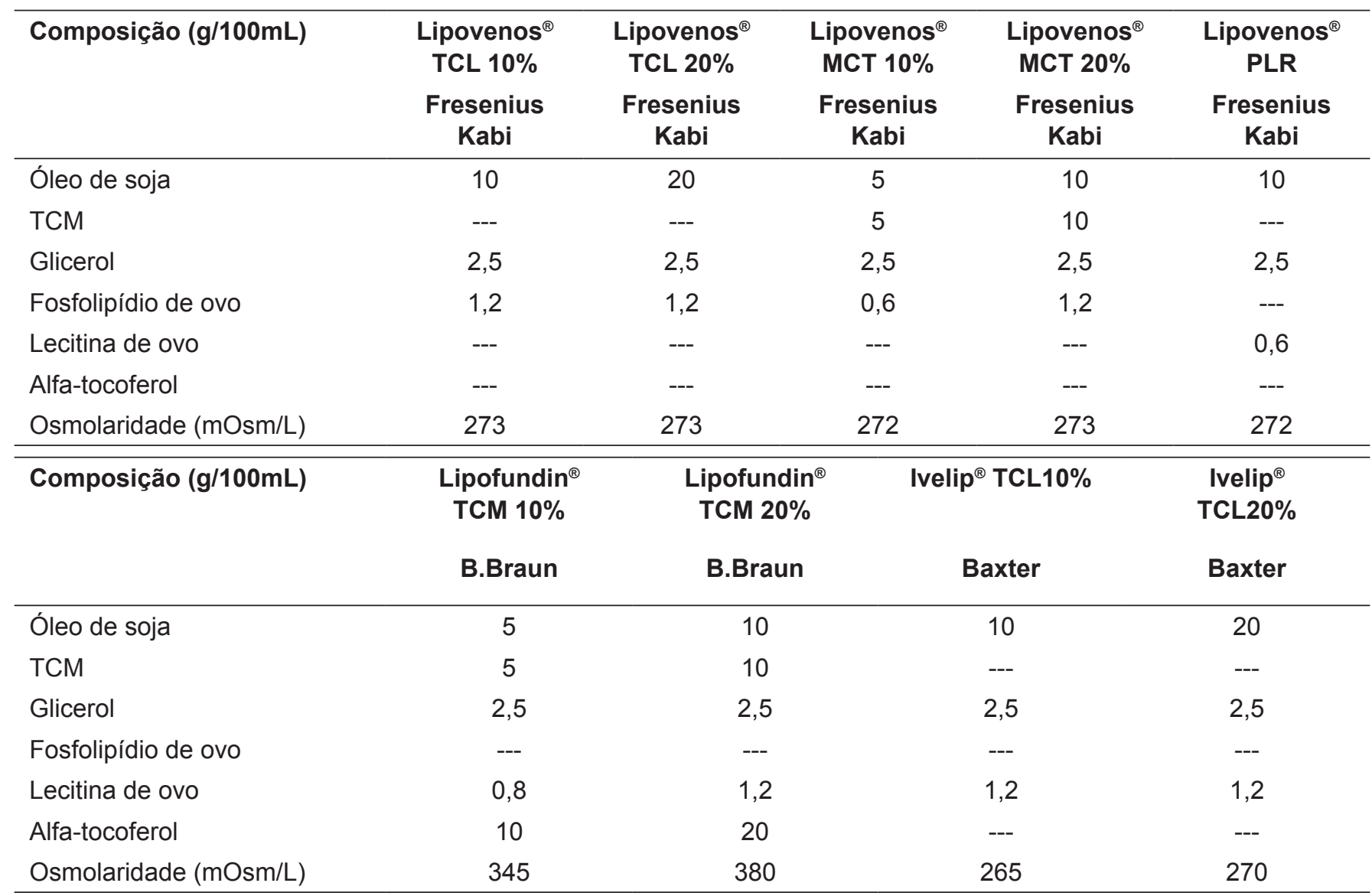

48s) a infusão e observar se há melhora do quadro ${ }^{(14,34)}$. Em relação à colestase, o jejum é um dos mais importantes fatores de risco para o seu desenvolvimento. Desta forma, a administração da nutrição enteral, mesmo com pequenos volumes, é fundamental ${ }^{(36)}$.

\section{Glicose}

A D-glicose (dextrose) é o carboidrato utilizado nas soluções de NP e fornece 3,4kcal/g. Soluções com concentração de 5, 10, 25 e 50\% estão disponíveis. A glicose desempenha papel fundamental para o metabolismo do sistema nervoso central. Para o cálculo da quantidade a ser acrescida na NP, utiliza-se a taxa de infusão ou velocidade de infusão de glicose (TIG ou VIG), expressa em $\mathrm{mg} / \mathrm{kg} / \mathrm{min}$. Geralmente, inicia-se com VIG de $4 \mathrm{mg} / \mathrm{kg} / \mathrm{min}$, progredindo-se até 12 e $14 \mathrm{mg} / \mathrm{kg} / \mathrm{min}$, dependendo das necessidades e condição clínica do neonato (Quadros 1 e 2). Nesta progressão, é fundamental monitorizar a glicemia, sendo o ideal valores entre 70 a $120 \mathrm{mg} / \mathrm{dL}^{(13,28,37)}$. Episódios de hipoglicemia estão associados com pior desenvolvimento neuropsicomotor futuro $^{(38)}$ e os episódios de hiperglicemia se relacionam ao maior número de complicações, especialmente de natureza infecciosa, e elevação no risco de mortalidade ${ }^{(39)}$.

\section{Vitaminas}

As vitaminas são adicionadas de forma combinada, em ampolas, na solução de NP. É importante conhecer a recomendação e a composição dos multivitamínicos disponíveis para ofertar as quantidades adequadas ${ }^{(40)}$, lembrando que não há produtos específicos para o RNPT. Um cálculo aproximado, levando em conta as recomendações para esta faixa etária e as soluções mais comumente utilizadas em nosso país, está disponível no algoritmo proposto ao final deste capítulo (Tabela 3, Figura 1).

Destaque especial pode ser dado à vitamina $\mathrm{A}$, pois as soluções disponíveis no mercado muitas vezes não atendem às necessidades do neonato, sem levar a um excesso no fornecimento de outras vitaminas. Uma metanálise recente concluiu que a suplementação de vitamina A (intramuscular, intravenosa ou por via oral) em RNPT com peso $<1500$ g está associada com redução da mortalidade e da necessidade de oxigenoterapia com um mês de vida. Para neonatos $<1000$ g, 
Quadro 1 - Parâmetros para acompanhamento da terapia nutricional parenteral do recém-nascido pré-termo.

\begin{tabular}{|c|c|c|}
\hline & Esperado & Significado \\
\hline $\begin{array}{l}\text { a) Concentração de cálcio } \\
\text { Ca (mEq) ------ volume na NP } \\
\text { x (mEq) ----- } 1000 \mathrm{~mL}\end{array}$ & $<10 \mathrm{mEq} / \mathrm{L}$ & $\begin{array}{l}\text { Trata-se da [Ca] em mEq/L na solução de NP. } \\
\text { Se }>10 \mathrm{mEq} / \mathrm{L} \text {, pode instabilizar a solução. }\end{array}$ \\
\hline $\begin{array}{l}\text { b) Somatória de cátions } \\
\mathrm{Ca}(\mathrm{mEq})+\mathrm{Mg}(\mathrm{mEq}) \text {---- volume na NP } \\
x(\mathrm{mEq}) \text { - }\end{array}$ & {$[\mathrm{Mg}+\mathrm{Ca}]<16 \mathrm{mEq} / \mathrm{L}$} & $\begin{array}{l}\text { Trata-se da somatória dos mEq dos cátions } \\
\text { (bi e trivalentes) na solução. Se }>16 \mathrm{mEq} / \mathrm{L} \text {, } \\
\text { pode instabilizar a solução. }\end{array}$ \\
\hline $\begin{array}{l}\text { c) Relação } \mathrm{Ca} / \mathrm{P}(\mathrm{mg}) \\
\mathrm{Ca}(\mathrm{mg}) \text { na NP } \\
\text { P }(\mathrm{mg}) \text { na NP }\end{array}$ & $\begin{array}{l}1,3: 1 \text { a } 2: 1(\mathrm{mg}) \\
1,1: 1 \text { a } 1,3: 1 \text { (molar) }\end{array}$ & $\begin{array}{l}\text { Trata-se da relação da quantidade de Ca } \\
\text { e P para manter a estabilidade da solução, } \\
\text { quando são utilizados sais inorgânicos de } \\
\text { fósforo. Nível próximo de } 2: 1 \text { (mg) é melhor } \\
\text { para incorporação óssea. }\end{array}$ \\
\hline $\begin{array}{l}\text { d) Osmolaridade (mOsm/L) } \\
\text { Osm=(AX8)+(GX7)+(Nax2)+(Px0,2)-50 } \\
\mathrm{G}=\mathrm{Glicose}(\mathrm{g} / \mathrm{L}) ; \mathrm{A}=\text { =aminoácidos }(\mathrm{g} / \mathrm{L}) ; \\
\text { Na=sódio }(\mathrm{mEq} / \mathrm{L} ; \mathrm{P}=\text { =fósforo }(\mathrm{mg} / \mathrm{L})\end{array}$ & $\begin{array}{l}<600 \mathrm{mOsm} / \mathrm{L} \\
\text { (periférico) } \\
>600 \mathrm{mOsm} / \mathrm{L} \\
\text { (central) }\end{array}$ & $\begin{array}{l}\text { É o melhor parâmetro para definir a via de } \\
\text { utilização da NP: se }<600 \text {, é seguro usar } \\
\text { via periférica; } 600-900 \text {, pode-se usar a via } \\
\text { periférica com cuidado; e }>900 \text {, é obrigatório } \\
\text { usar a via central . }\end{array}$ \\
\hline $\begin{array}{l}\text { e) Kcal não protéicas/grama } \mathrm{N}(\mathrm{C} / \mathrm{N}) \\
\mathrm{N} / \mathrm{C}=\mathrm{g} \mathrm{N} / \mathrm{kcal} \text { Lipídio+kcal (glicose) } \\
1 \mathrm{~g} \text { proteína }=0,16 \mathrm{~g} \text { nitrogênio }(4 \mathrm{kcal}) \\
1 \mathrm{~g} \text { lipídio }=9 \mathrm{kcal} \\
\text { 1g glicose }=3,4 \mathrm{kcal}\end{array}$ & $\begin{array}{l}\text { 100/1-150/1 } \\
\text { (estresse metabólico) } \\
\text { 150/1-250/1 } \\
\text { (para anabolismo) }\end{array}$ & $\begin{array}{l}\text { Caracterização da distribuição dos nutrientes. } \\
\text { RN hipercatabólicos ou em estresse } \\
\text { metabólico beneficiam-se da relação de } \\
\text { 100/1-150/1. RN em anabolismo necessitam } \\
\text { de relações de 150/1-250/1. Tal relação não é } \\
\text { tão importante no RNPT. }\end{array}$ \\
\hline $\begin{array}{l}\text { f) Concentração de glicose } \\
\text { [Glicose]=g (glicose)/volume total }\end{array}$ & $>12,5 \%$ & $\begin{array}{l}\text { Não pode ser o único parâmetro para definir } \\
\text { se a solução será utilizada na via periférica } \\
\text { ou central. }\end{array}$ \\
\hline $\begin{array}{l}\text { g) Oferta calórica } \\
\text { OC=kcal (lipídios)+kcal(proteínas)+kcal } \\
\text { (glicose)/peso }(\mathrm{kg})\end{array}$ & Calorias da NP/peso & $\begin{array}{l}\text { Cálculo para verificar se as calorias } \\
\text { necessárias estão sendo oferecidas para } \\
\text { síntese/manutenção dos órgãos, tecidos e } \\
\text { sistemas. }\end{array}$ \\
\hline
\end{tabular}

tais achados também foram observados após 36 semanas de idade gestacional corrigida ${ }^{(14,41)}$.

Em situações nas quais há necessidade de emprego exclusivo de NP, sem utilização da via enteral ou na presença de colestase ou sepse, pode-se realizar suplementação intravenosa ou intramuscular com vitamina $\mathrm{K}$ na dose de 1 a $3 \mathrm{mg} / \mathrm{semana}$.

Outro cuidado importante que reduz as perdas vitamínicas é o uso de frascos e equipos com fotoproteção. A exposição à luz leva à degradação das vitaminas fotossensíveis e a uma maior produção de peróxidos lipídicos na $\mathrm{NP}^{(42)}$. Um trabalho recente sugere que a fotoproteção da solução de NP reduziria em $30 \%$ a incidência de doença pulmonar crônica em RNPT ${ }^{(43)}$.

\section{Oligoelementos}

De maneira similar às vitaminas, os oligoelementos são adicionados à solução de NP de forma combinada, em ampolas, e também não há produtos específicos para $\mathrm{RNPT}^{(40,44)}$ (Tabela 4). Uma sugestão de oferta, baseada nas recomendações e composição das soluções disponíveis no mercado, é apresentada na Figura 1. Há soluções disponíveis apenas 
Quadro 2 - Controles clínicos e laboratoriais sugeridos na terapia nutricional do recém-nascido pré-temo.

\begin{tabular}{|lcc|}
\hline Parâmetros & Período inicial & Período estável \\
\hline $\begin{array}{l}\text { Clínicos (atividade, diurese, pressão arterial, } \\
\text { freqüência cardíaca, freqüência respiratória, }\end{array}$ & Diariamente & Diariamente \\
temperatura, balanço hídrico) & & \\
Eletrólitos e pH (Na, K, Ca, P, Mg) & $3-4 x$ semana & Semanal \\
Uréia e creatinina & $2-3 x$ semana & Semanal \\
Glicemia (dextro) & Diariamente (3 vezes) & Diariamente (1 vez) \\
Glicosúria & Diariamente (3 vezes) & Diariamente (1 vez) \\
Albumina & Semanal & Semanal \\
Enzimas hepáticas (TGO, TGP, Gama-GT) & Semanal & Semanal \\
Hemoglobina e hematócrito & Semanal & Semanal \\
Glicosúria & Diariamente & Diariamente \\
Densidade urinária & Diariamente & Diariamente \\
Leucograma & Quando indicado & Quando indicado \\
Culturas & Quando indicado & Quando indicado \\
Triglicérides (não é necessário colesterol total e frações) & 4 horas após $\Uparrow$ infusão lípides & Semanal \\
\hline
\end{tabular}

Tabela 3 - Necessidades vitaminas lipo e hidrossolúveis kg/dia em recém-nascido pré-termo e composição para cada mL das soluções

\begin{tabular}{|c|c|c|c|c|c|}
\hline & $\begin{array}{l}\text { Recomendação } \\
\text { diária (kg/dia) }\end{array}$ & $\begin{array}{c}\text { M.V.I } 12 \\
\text { Opoplex } \\
\text { Pediátrico } \\
\text { ampola } 5 \mathrm{~mL} \\
\text { Grossman } \\
\text { Para cada } 1 \mathrm{~mL}\end{array}$ & $\begin{array}{c}\text { Polivit } ® A \\
\text { Pediátrico } \\
\text { ampola } 10 \mathrm{~mL} \\
\text { Inpharma } \\
\text { Para cada } 1 \mathrm{~mL}\end{array}$ & $\begin{array}{c}\text { Polivit }^{\circledR} \text { B } \\
\text { Pediátrico } \\
\text { ampola 5mL } \\
\text { Inpharma } \\
\text { Para cada 1mL }\end{array}$ & $\begin{array}{c}\text { Cristália } \\
\text { Para cada } 1 \mathrm{~mL}\end{array}$ \\
\hline \multicolumn{6}{|l|}{ Vitaminas lipossolúveis } \\
\hline$A(\mu g)$ & 700 & 122 & 70 & --- & 304 \\
\hline $\mathrm{E}(\mathrm{mg})$ & 2,8 & 1,4 & 0,7 & --- & 5 \\
\hline $\mathrm{K}(\mu \mathrm{g})$ & 80 & 40 & --- & --- & --- \\
\hline$D(\mu g)$ & 4 & 1 & 1 & --- & 2 \\
\hline \multicolumn{6}{|c|}{ Vitaminas hidrossolúveis } \\
\hline Ácido ascórbico (mg) & 25 & 16 & 8 & --- & 50 \\
\hline Tiamina $(\mathrm{mg})$ & 0,35 & 0,3 & 0,12 & --- & --- \\
\hline Riboflavina (mg) & 0,15 & 0,35 & 0,14 & --- & 0,5 \\
\hline Piridoxina (mg) & 0,18 & 0,25 & 0,10 & --- & 1,5 \\
\hline Niacina (mg) & 6,8 & 4,25 & 1,7 & --- & 10 \\
\hline Ácido patotênico (mg) & 2,0 & 1,25 & 0,5 & --- & 2,5 \\
\hline Biotina $(\mu \mathrm{g})$ & 6,0 & 4 & --- & 4 & --- \\
\hline Folato $(\mu \mathrm{g})$ & 56,0 & 28 & --- & 35 & --- \\
\hline Vitamina B12 $(\mu \mathrm{g})$ & 0,3 & 0,2 & --- & 0,2 & --- \\
\hline Dose/dia & & $2 \mathrm{~mL} / \mathrm{kg}$ & $4 \mathrm{~mL} / \mathrm{kg}$ & $2 \mathrm{~mL} / \mathrm{kg}$ & $2 \mathrm{~mL} / \mathrm{kg}$ \\
\hline
\end{tabular}

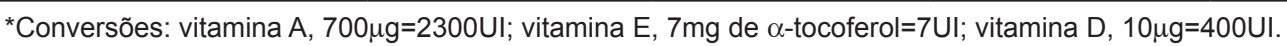


Tabela 4 - Necessidades de oligoelementos (kg/dia) em recém-nascidos pré-termo e composição para cada $\mathrm{mL}$ das soluções de oligoelementos

\begin{tabular}{|c|c|c|c|c|c|c|}
\hline $\begin{array}{l}\text { Nutriente } \\
\text { Fabricante }\end{array}$ & $\begin{array}{c}\text { Recomendação } \\
(\mu \mathrm{g} / \mathrm{kg} / \mathrm{dia})\end{array}$ & $\begin{array}{c}\text { Ped-Element }^{\circledR} \\
\text { (ampola } 5 \mathrm{~mL} \text { ) } \\
\text { Darrow } \\
(\mu \mathrm{g} / \mathrm{mL} \text { ) }\end{array}$ & 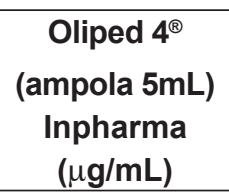 & $\begin{array}{c}\text { Ad-ELement }^{\circledR} \\
\text { (ampola } 2 \mathrm{~mL} \text { ) } \\
\text { Darrow } \\
(\mu \mathrm{g} / \mathrm{mL})\end{array}$ & $\begin{array}{c}\text { Politrace }^{\circledR} 4 \\
\text { (ampola } 5 \mathrm{~mL} \text { ) } \\
\text { Inpharma } \\
(\mu \mathrm{g} / \mathrm{mL})\end{array}$ & $\begin{array}{c}\text { Tracitrans }^{\circledR} \text { Plus }^{*} \\
\text { (ampola 10mL) } \\
\text { Fresenius } \\
(\mu \mathrm{g} / \mathrm{mL})\end{array}$ \\
\hline Zinco $(\mu \mathrm{g})$ & 400 & 500 & 100 & 1250 & 1000 & 660 \\
\hline Cobre $(\mu \mathrm{g})$ & 20 & 100 & 20 & 400 & 200 & 127 \\
\hline Selênio $(\mu \mathrm{g})$ & 2,0 & --- & --- & --- & --- & 3,2 \\
\hline Cromo $(\mu \mathrm{g})$ & 0,20 & 1 & 0,17 & 5 & 2 & 1 \\
\hline Manganês ( $\mu \mathrm{g})$ & 1,0 & 10 & 6 & 200 & 100 & 27 \\
\hline Molibdênio ( $\mu \mathrm{g})$ & 0,25 & --- & --- & --- & --- & 2 \\
\hline lodo $(\mu \mathrm{g})$ & 1,0 & --- & --- & --- & --- & 13 \\
\hline Dose/dia \& & & $0,2 \mathrm{~mL} / \mathrm{kg}$ & $1 \mathrm{~mL} / \mathrm{kg}$ & $0,05 \mathrm{~mL} / \mathrm{kg}^{\#}$ & $0,1 \mathrm{~mL} / \mathrm{kg}$ & $0,3 \mathrm{~mL} / \mathrm{kg}$ \\
\hline
\end{tabular}

${ }^{*}$ Tracitrans ${ }^{\circledR}$ plus: contém também ferro $112 \mu \mathrm{g} / \mathrm{mL}$ e flúor $95 \mu \mathrm{g} / \mathrm{mL}$; \#Mesmo com o ajuste da dose, a oferta de manganês fica dez vezes acima do recomendado por dia; \&O cálculo foi baseado na recomendação de cobre e, por isso, alguns oligoelementos ficam acima e outros abaixo do recomendado, uma vez que não é possível o calculo individualizado. No caso do zinco, há solução individualizada e é necessário acrescentar 200 a $400 \mu \mathrm{g}$ além do oferecido pela solução de oligoelementos para ajuste das necessidades.

com zinco na sua composição $(200 \mu \mathrm{g} / \mathrm{mL}$ e $1.000 \mu \mathrm{g} / \mathrm{mL})$, que podem ser utilizadas em combinação com as soluções de oligoelementos para suprir as necessidades extras de zinco neste faixa etária.

Alguns cuidados são importantes: o manganês e o cobre são de excreção hepática. Em condições em que há prejuízo da excreção da bile (colestase), pode-se reduzir ou até excluir a adição destes oligoelementos na NP. É possível, mesmo com a NP exclusiva, usar apenas a solução de zinco por período de duas semanas, sem prejuízo para o RNPT ${ }^{(14,44)}$.

Estudos recentes indicam ser o selênio um importante oligoelemento na terapia nutricional do RNPT desde os primeiros dias de vida, especialmente para reduzir os episódios de sepse ${ }^{(45,46)}$. Infelizmente, as soluções pediátricas de oligoelementos disponíveis no mercado não contêm selênio. Uma opção para suplementar o selênio é administrar a solução de oligoelementos para adultos (adaptando as quantidades a serem oferecidas pela Tracitrans ${ }^{\circledR}$ Plus) ou a alimentação suplementar por via via enteral.

\section{Minerais}

Um dos grandes desafios da terapia nutricional do RNPT é oferecer grandes quantidades de cálcio e fósforo, com relação adequada para a mineralização óssea (relação cálcio:fósforo de 1,3:1 a 2:1 mg ou de 1,1:1 a 1,3:1 na relação molar) e sem levar à precipitação da solução. Para facilitar a administração, prefere-se usar o fósforo na forma orgânica (Tabela 5). As necessidades por via parenteral são de 80 a $100 \mathrm{mg} / \mathrm{kg} / \mathrm{dia}$ de cálcio e 43 a $62 \mathrm{mg} / \mathrm{kg} / \mathrm{dia}$ de fósforo ${ }^{(40)}$.
Os outros eletrólitos (sódio, potássio, magnésio) são acrescidos à solução segundo os dias de vida e as quantidades recomendadas (Figura 1). Alguns cuidados devem ser considerados na oferta de eletrólitos nas soluções $3: 1^{(44)}$ : a quantidade de cálcio na solução não deve ultrapassar $10 \mathrm{mEq} / \mathrm{L}$ e a somatória dos cátions di ou trivalentes (cálcio e magnésio) deve permanecer abaixo de $16 \mathrm{mEq} / \mathrm{L}$ (Quadro 1).

A oferta hídrica inicial deve ser de $80 \mathrm{~mL} / \mathrm{kg} / \mathrm{dia}$ para $\mathrm{RN}$ $<1.000 \mathrm{~g} \mathrm{e} 70 \mathrm{~mL} / \mathrm{kg}$ para os demais ${ }^{(13)}$. A progressão do volume deve levar em conta o balanço hídrico e outros líquidos infundidos via enteral ou parenteral, sendo que, ao término da primeira semana de vida, deve estar em torno de 130 a $150 \mathrm{~mL} / \mathrm{kg} /$ dia. É importante também considerar, para a oferta hídrica, a presença de doenças que levam à necessidade de restrição hídrica, como a persistência do canal arterial.

\section{Nutrição enteral}

A nutrição enteral deve ser introduzida precocemente, preferencialmente nas primeiras 48 horas de vida, em pequenas quantidades, com leite humano cru ( $1^{\mathrm{a}}$ opção) ou pasteurizado ( $2^{\mathrm{a}}$ opção). A progressão é feita, dependendo da condição clínica e da tolerância da dieta pelo RNPT, com incrementos de 20 a $30 \mathrm{~mL} / \mathrm{kg} / \mathrm{dia}^{(4,17)}$, porém deve-se lembrar que o uso de leite humano se associa à melhor tolerância e, portanto, pode-se efetuar progressão mais rápida da dieta por via enteral. $\mathrm{Na}$ impossibilidade de utilizar leite materno, a opção mais apropriada é o emprego de fórmulas infantis específicas para o pré-termo. 
Tabela 5 - Composição das soluções de eletrólitos

\begin{tabular}{|c|c|c|c|c|}
\hline \multirow[t]{2}{*}{ Produto } & \multicolumn{2}{|c|}{ Cátion } & \multicolumn{2}{|c|}{ Ânion } \\
\hline & $\mathrm{mEq} / \mathrm{mL}$ & $\mathrm{mg} / \mathrm{mL}$ & $\mathrm{mEq} / \mathrm{mL}$ & $\mathrm{mg} / \mathrm{mL}$ \\
\hline Acetato de sódio $10 \%$ & 0,7 & 16,9 & 0,7 & 43,4 \\
\hline Acetato de sódio $2 \mathrm{mEq} / \mathrm{mL}$ & 2,0 & 46,0 & 2,0 & 118,1 \\
\hline Acetato de potássio $2 \mathrm{mEq} / \mathrm{mL}$ & 2,0 & 78,2 & 2,0 & 117,9 \\
\hline Bicarbonato de sódio $10 \%$ & 1,2 & 27,4 & 1,2 & 72,6 \\
\hline Cloreto de sódio $3 \%$ & 0,5 & 13,1 & 0,5 & 20,2 \\
\hline Cloreto de sódio $10 \%$ & 1,7 & 39,3 & 1,7 & 60,7 \\
\hline Cloreto de sódio $20 \%$ & 3,4 & 78,6 & 3,4 & 121,4 \\
\hline Cloreto de sódio $4 \mathrm{mEq} / \mathrm{mL}$ & 4,0 & 92 & 4,0 & 141,8 \\
\hline Cloreto de potássio $19,1 \%$ & 2,6 & 100,1 & 2,6 & 90,9 \\
\hline Cloreto de potássio $2 \mathrm{mEq} / \mathrm{mL}$ & 2,0 & 78,2 & 2,0 & 70,9 \\
\hline Fosfato diácido de potássio $25 \%$ & 1,8 & 71,8 & 1,8 & 57,0 \\
\hline Fosfato monoácido de potássio $25 \%$ & 2,9 & 112,3 & 1,9 & 44,5 \\
\hline Fosfato de Sódio $2 \mathrm{mEq} / \mathrm{mL}$ & 2,0 & 46,0 & $1,1^{*}$ & 34,2 \\
\hline Fosfato de potássio $2 \mathrm{mEq} / \mathrm{mL}$ & 2,0 & 96 & $1,1^{*}$ & 34,2 \\
\hline Glicerofosfato de sódio $1 \mathrm{Mmol} / \mathrm{mL}$ & $2,0^{*}(\mathrm{Na})$ & 46 & $1,0^{*}$ & 31,0 \\
\hline Glicerofosfato de sódio $0,33 \mathrm{Mmol} / \mathrm{mL}$ & $0,66^{*}(\mathrm{Na})$ & 15,3 & $0,33^{*}$ & 10,2 \\
\hline Gluconato de cálcio $10 \%$ & 0,5 & 8,9 & 0,5 & 87,0 \\
\hline Sulfato de magnésio $10 \%$ & 0,8 & 9,9 & 0,8 & 39 \\
\hline Sulfato de magnésio $20 \%$ & 1,6 & 19,8 & 1,6 & 78,0 \\
\hline Sulfato de magnésio $50 \%$ & 4,0 & 49,5 & 4,0 & 195,0 \\
\hline Sulfato de magnésio $1 \mathrm{mEq} / \mathrm{mL}$ & 1,0 & 12,2 & 1,0 & 48,1 \\
\hline
\end{tabular}

${ }^{*} \mathrm{Mmol} / \mathrm{L}$

\section{Monitorização}

A implantação da estratégia de seguimento e monitorização da utilização de NP é fundamental para prevenir e tratar precocemente as complicações relacionadas ao seu uso. A participação de equipe multidisciplinar na instituição de protocolos de cuidados com cateter, de manipulação, interação droga-nutriente, instalação, fotoproteção, avaliação nutricional e seguimento metabólico e laboratorial aumentam a segurança e a efetividade da utilização da NP em RNPT (Quadro 1 e 2).

\section{Complicações}

As complicações mais graves associadas à utilização da NP em RNPT são as hepatobiliares (colestase) e a sepse ${ }^{(47)}$. Protocolos de assistência e monitorização do uso de NP, com o envolvimento da equipe multidisciplinar, previnem e reduzem de forma significativa as complicações associadas à $\mathrm{NP}^{(9)}$. Medidas como cuidados com a preparação da solução, posicionamento e manutenção adequados dos cateteres diminuem sobremaneira as complicações infecciosas relacionadas à NP.

Para a colestase do RNPT associada a NP, os fatores de risco mais importantes são o tempo de jejum enteral, o tempo de utilização da NP e a sepse. Uma vez instalada a colestase, devese considerar, para o seu tratamento, a utilização de nutrição enteral (mesmo em pequenos volumes), a redução da oferta de aminoácidos e da oferta de glicose, a manutenção dos níveis de triglicérides circulantes abaixo de $200 \mathrm{mg} / \mathrm{dL}$ (se necessário, suspender a infusão lipídica por 24 a 48 horas), a suspensão temporária (sete a 14 dias) da solução de oligoelementos, visto ser o cobre e o manganês de excreção hepática. Em casos mais avançados, pode-se utilizar a NP cíclica ${ }^{(14,47)}$.

\section{Considerações finais}

Esse protocolo prático sobre NP para RNPT foi desenvolvido com base em recomendações internacionais de sociedades científicas e a análise crítica de trabalhos científicos 
com metodologia apropriada abordando o tema. $\mathrm{O}$ artigo pretende facilitar o treinamento de residentes de pediatria e embasar o desenvolvimento de protocolos por equipes multiprofissionais de terapia nutricional.

\section{Referências bibliográficas}

1. Ehrenkranz RA. Early, aggressive nutritional management for very low birth weight infants: what is the evidence? Semin Perinatol 2007;31:48-55.

2. Bertino E, Coscia A, Mombrò M, Boni L, Rossetti G, Fabris C et al. Postnatal weight increase and growth velocity of very low birthweight infants. Arch Dis Child Fetal Neonatal Ed 2006;91:F349-56.

3. Ehrenkranz RA, DusickAM, Vohr BR, Wright LL, Wrage LA, Poole WK. Growth in the neonatal intensive care unit influences neurodevelopmental and growth outcomes of extremely low birth weight infants. Pediatrics 2006;117:1253-61.

4. Sangild PT. Gut responses to enteral nutrition in preterm infants and animals. Exp Biol Med (Maywood) 2006;231:1695-711.

5. Thureen PJ, Hay WW Jr. Early aggressive nutrition in preterm infants. Semin Neonatol 2001;6:403-15.

6. Demmelmair H, von Rosen J, Koletzko B. Long-term consequences of early nutrition. Early Hum Dev 2006;82:567-74.

7. Chowdhary SK, Parashar K. Central venous access in neonates through the peripheral route. Curr Opin Clin Nutr Metab Care 2000;3:217-9.

8. Racadio JM, Johnson ND, Doellman DA. Peripherally inserted central venous catheters: success of scalp-vein access in infants and newborns. Radiology 1999;210:858-60.

9. Agostoni C, Axelson I, Colomb V, Goulet O, Koletzko B, Michaelsen KF et al. The need for nutrition support teams in pediatric units: a commentary by the ESPGHAN committee on nutrition. J Pediatr Gastroenterol Nutr 2005;41:8-11 .

10. Forbes A. Parenteral nutrition. Curr Opin Gastroenterol 2007;23:183-6.

11. Brasil - Ministério da Saúde. Média e Alta Complexidade. Aprova o Regulamento Técnico para fixar os requisitos mínimos exigidos para a Terapia de Nutrição Parenteral. Portaria n 272/MS/SNVS, 1998.

12. Brasil - Ministério da Saúde. Média e Alta Complexidade. Define Unidades de Assistência de Alta Complexidade em Terapia Nutricional e Centros de Referência de Alta Complexidade em Terapia Nutricional e suas aptidões e qualidades. Portaria $n^{\circ}$ 131/MS/SNVS, 2005.

13. American Academy of Pediatrics, Committee on Nutrition (AAP-CON). Pediatric nutrition handbook. Elk Grove Village: American Academy of Pediatrics; 2003.

14. ASPEN Board of Directors and the Clinical Guidelines Task Force. Guidelines for the use of parenteral and enteral nutrition in adult and pediatric patients. JPEN J Parenter Enteral Nutr 2002;26(1 Suppl):1SA-138SA.

15. Koletzko B, Goulet O, Hunt J, Krohn K, Shamir R: Parenteral Nutrition Guidelines Working Group; European Society for Clinical Nutrition and Metabolism; European Society of Paediatric Gastroenterology, Hepatology and Nutrition (ESPGHAN); European Society of Paediatric Research (ESPR). Guidelines on paediatric parenteral nutrition of the European Society of Paediatric Gastroenterology, Hepatology and Nutrition (ESPGHAN) and the European Society for Clinical Nutrition and Metabolism (ESPEN), supported by the European Society of Paediatric Research (ESPR). J Pediatr Gastroenterol Nutr 2005;41(Suppl 2): $\$ 1-87$

16. Heird WC. Determination of nutritional requirements in preterm infants, with special reference to 'catch-up' growth. Semin Neonatol 2001;6:365-75.

17. Neu J. Gastrointestinal development and meeting the nutritional needs of premature infants. Am J Clin Nutr 2007;85:629S-634S.

18. Hay WW Jr, Thureen PJ. Early postnatal administration of intravenous amino acids to preterm, extremely low birth weight infants. J Pediatr 2006;148:291-4.

19. Ziegler EE, Thureen PJ, Carlson SJ. Aggressive nutrition of the very low birthweight infant. Clin Perinatol 2002;29:225-44.

20. Greer FR. Post-discharge nutrition: what does the evidence support? Semin Perinatol 2007;31:89-95.

21. Kuschel CA, Harding JE. Multicomponent fortified human milk for promoting growth in preterm infants. Cochrane Database Syst Rev 2004:CD000343.
22. Boyd CA, Quigley MA, Brocklehurst P. Donor breast milk versus infant formula for preterm infants: systematic review and meta-analysis. Arch Dis Child Fetal Neonatal Ed 2007;92:F169-75.

23. Espen/ESPGHAN Guidelines on paediatric parenteral nutrition. Venous access. J Pediatr Gastroenterol Nutr 2005;41:S54-62.

24. Kalhan SC, Iben S. Protein metabolism in the extremely low-birth weight infant. Clin Perinatol 2000;27:23-56.

25. Thureen PJ, Melara D, Fennessey PV, Hay WW Jr. Effect of low versus high intravenous amino acid intake on very low birth weight infants in the early neonatal period. Pediatr Res 2003;53:24-32.

26. Ibrahim HM, Jeroudi MA, Baier RJ, Dhanireddy R, Krouskop RW. Aggressive early total parental nutrition in low-birth-weight infants. J Perinatol 2004;24:482-6.

27. Simmer K. Aggressive nutrition for preterm infants-benefits and risks. Early Hum Dev 2007;83:631-4.

28. Farrag HM, Cowett RM. Glucose homeostasis in the micropremie. Clin Perinatol 2000;27:1-22

29. Espen/ESPGHAN Guidelines on paediatric parenteral nutrition. Amino acids. J Pediatr Gastroenterol Nutr 2006;41:S12-8.

30. Riedijk MA, van Beek RH, Voortman G, de Bie HM, Dassel AC, van Goudoever JB. Cysteine: a conditionally essential amino acid in low-birth-weight preterm infants? Am J Clin Nutr 2007:86:1120-5.

31. Tubman TR, Thompson SW, McGuire W. Glutamine supplementation to prevent morbidity and mortality in preterm infants. Cochrane Database Syst Rev 2005;25:CD001457.

32. Wanten GJ, Calder PC. Immune modulation by parenteral lipid emulsions. Am J Clin Nutr 2007;85:1171-84.

33. Krohn K, Koletzko B. Parenteral lipid emulsions in paediatrics. Curr Opin Clin Nutr Metab Care 2006;9:319-23.

34. Espen/ESPGHAN Guidelines on paediatric parenteral nutrition. Lipids. J Pediatr Gastroenterol Nutr 2006;41:S19-27.

35. Shah PS, Kalyn A, Satodia P, Dunn MS, Parvez B, Daneman A et al. A randomized, controlled trial of heparin versus placebo infusion to prolong the usability of peripherally placed percutaneous central venous catheters (PCVCs) in neonates: the HIP (Heparin Infusion for PCVC) study. Pediatrics 2007;119:e284-91.

36. Robinson DT, Ehrenkranz RA. Parenteral nutrition-associated cholestasis in small for gestational age infants. J Pediatr 2008;152:59-62.

37. Espen/ESPGHAN Guidelines on paediatric parenteral nutrition. Carbohidrates. J Pediatr Gastroenterol Nutr 2006;41:S28-32.

38. Kinnala A, Nuutila P, Ruotsalainen U, Teräs M, Bergman J, Haaparanta M et al. Cerebral metabolic rate for glucose after neonatal hypoglycaemia. Early Hum Dev 1997;49:63-72.

39. Heimann K, Peschgens T, Kwiecien R, Stanzel S, Hoernchen H, Merz U. Are recurrent hyperglycemic episodes and median blood glucose level a prognostic factor for increased morbidity and mortality in premature infants $\leq 1500 \mathrm{~g}$ ? J Perinat Med 2007;35:245-8.

40. Greene HL, Hambidge KM, Schanler R, Tsang RC. Guidelines for the use of vitamins, trace elements, calcium, magnesium, and phosphorus in infants and children receiving total parenteral nutrition: report of the Subcommittee on Pediatric Parenteral Nutrient Requirements from the Committee on Clinical Practice Issues of the American Society for Clinical Nutrition. Am J Clin Nutr 1988;48:1324-42.

41. Darlow BA, Graham PJ. Vitamin A supplementation to prevent mortality and short and long-term morbidity in very low birthweight infants. Cochrane Database Syst Rev 2007;17:CD000501.

42. Laborie S, Lavoie JC, Pineault M, Chessex P. Contribution of multivitamins, air, and light in the generation of peroxides in adult and neonatal parenteral nutrition solutions. Ann Pharmacother 2000;34:440-5. 
43. Chessex P, Harrison A, Khashu M, Lavoie JC. In preterm neonates, is the risk of developing bronchopulmonary dysplasia influenced by the failure to protect total parenteral nutrition from exposure to ambient light? J Pediatr 2007;151:213-4.

44. Espen/ESPGHAN Guidelines on paediatric parenteral nutrition. Iron, minerals and trace elements. J Pediatr Gastroenterol Nutr 2006;41:S39-46.

45. Darlow BA, Austin NC. Selenium supplementation to prevent short-term mor- bidity in preterm neonates. Cochrane Database Syst Rev 2003:CD003312.

46. Mentro AM, Smith AM, Moyer-Mileur L. Plasma and erythrocyte selenium and glutathione peroxidase activity in preterm infants at risk for bronchopulmonary dysplasia. Biol Trace Elem Res 2005;106:97-106.

47. Espen/ESPGHAN Guidelines on paediatric parenteral nutrition. Complications. J Pediatr Gastroenterol Nutr 2006;41:S76-84. 